\title{
Multiple intracerebral lesions in a young male
}

\author{
Madi Deepak ${ }^{1}$, Achappa Basavaprabhu ${ }^{1}$, Mahalingam Soundarya ${ }^{2}$
}

1. Kasturba Medical College, General Medicine, Manipal University

2. Kasturba Medical College, Paediatrics, Manipal University

\begin{abstract} difficult in resource limited settings. which showed significant resolution of the lesions.

DOI: http://dx.doi.org/10.4314/ahs.v15i3.47 doi: http://dx.doi.org/10.4314/ahs.v15i3.47

\section{Introduction}

Toxoplasmosis, Tuberculosis, cryptococcosis, CNS lymphoma and progressive multifocal leukoencephalopathy affect the central nervous system in HIV-infected patients. An accurate diagnosis of these neurological complications is crucial because effective intervention will lead to longer survival .

Toxoplasmosis occurs in patients with CD4 $\mathrm{T}$ cell counts below 200 cells/ $\mu \mathrm{L}$. Like most CNS diseases in HIV, establishing a definitive diagnosis of Toxoplasmosis is often difficult in resource limited settings thereby requiring a therapeutic trial ${ }^{1}$. In routine clinical practice treatment is usually initiated based on radiological features and serology.We present a case of CNS toxoplasmosis in a 20 year old male.
\end{abstract}

Background: As the incidence of HIV infection has increased its neurological complications are being encountered in our clinical practice. Toxoplasmosis is a common cerebral opportunistic infection seen in HIV-infected patients, even though the incidence has declined with the use of antiretroviral therapy. Establishing a definitive diagnosis of cerebral toxoplasmosis is

Clinical case: A 20 year old gentleman was referred to our institute as a case of stroke. Magnetic resonance imaging (MRI) of his brain showed multiple ill-defined and nodular enhancing lesions in bilateral supratentorial and infratentorial neuroparenchyma. Test for HIV-1 was reactive. Toxoplasma serology revealed raised IgG antibody levels. Based on the MRI features and positive toxoplasma serology a diagnosis of cerebral toxoplasmosis was made. He was treated with trimethoprim/ sulfamethoxazole and pyrimethamine/ Sulfadoxine for 3 weeks. After 2 weeks of treatment, repeat MRI of brain was done

Conclusion: We are presenting this case to highlight the fact that cerebral toxoplasmosis should be considered in the differential diagnosis of multiple neuroparenchymal lesions in young individuals who present with neurological deficits.

Keywords: Cerebral toxoplasmosis; HIV/AIDS; Tuberculoma; Neurocysticercosis

Cite as: Deepak. M, Basavaprabhu A, Soundarya M. Multiple intracerebral lesions in a young male. Afri Health Sci. 2015;15(3):1045-8.

\section{Case Report}

A 20-year-old male was referred to our institution as a

\section{Corresponding author:}

Achappa Basavaprabhu

Kasturba Medical College, General Medicine

Email: bachu1504@gmail.com;

bachu1504@yahoo.co.in case of stroke. He had suffered from mild headache for two weeks. One day prior to admission he developed confusion and right sided hemiparesis. He was treated for pulmonary tuberculosis 1 year ago. On examination he was confused and had right hemiparesis.

Lab investigations showed haemoglobin $-10.8 \mathrm{~g} / \mathrm{dl}$, total White Blood Cell count -5600 cells/cu.mm, Neutrophil- 86\%, Lymphocyte- 4\%,eosinophil- $6 \%$,Monocyte$4 \%$, platelet count-2,02,000 cells/cu.mm, ESR- $90 \mathrm{~mm}$. Serum electrolytes, blood sugar, renal and liver function tests were normal. Test for HIV-1 was reactive. His CD4T cell count was 22cells/ $\mu \mathrm{L}$. Chest X-ray and ultrasound of the abdomen were normal.

MRI brain (Figure 1) showed multiple ill-defined and nodular enhancing lesions in bilateral supratentorial and infratentorial neuroparenchyma. Involvement of bilateral basal ganglia, thalamus and brainstem was noted. Gram stain, Ziehl Neelsen stain, India ink stain and culture of CSF were negative. 


\section{Coronal T1weighted post contrast image showing supratentorial and infratentorial ring enhancing and nodular enhancing lesions on both sides.}

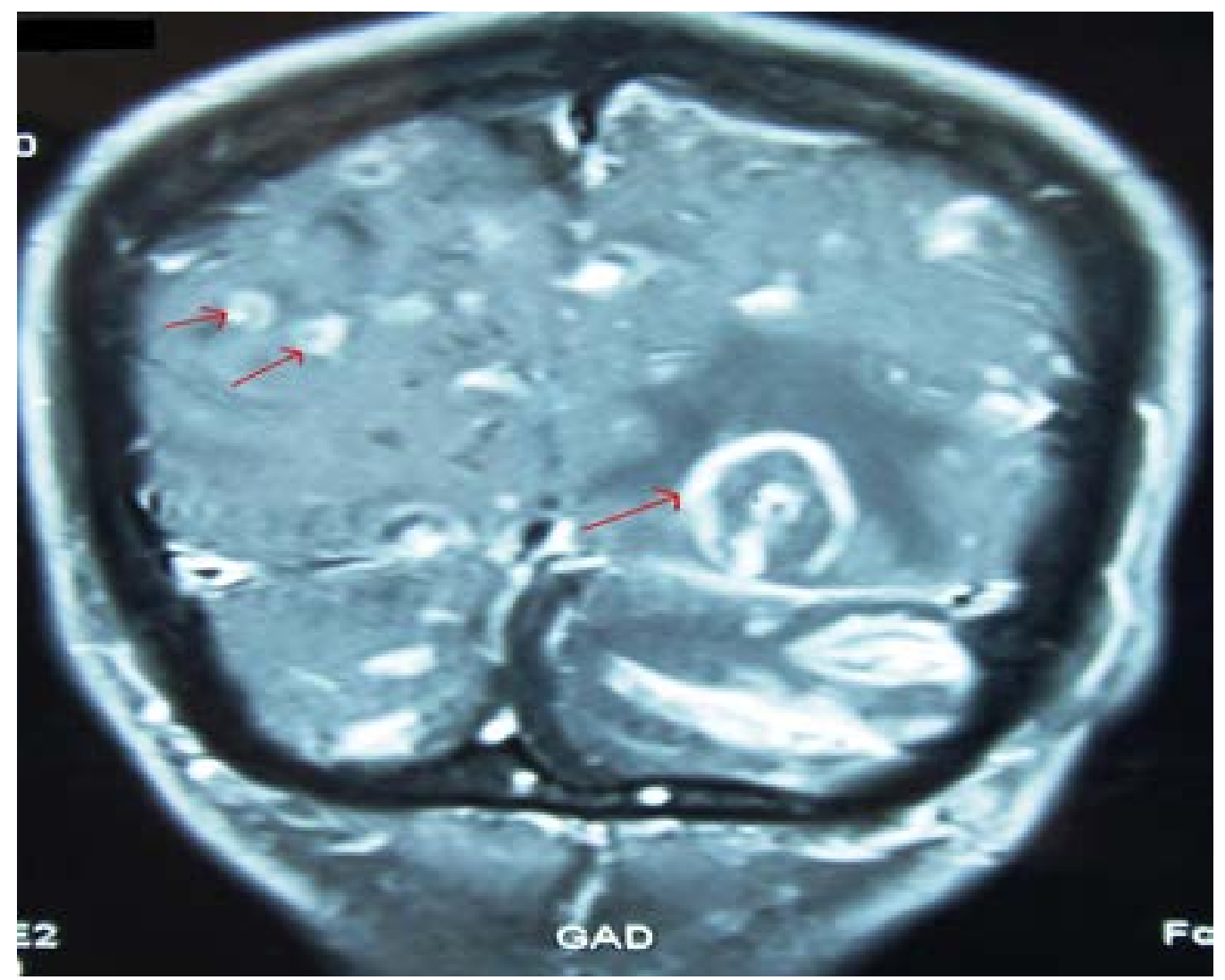

Toxoplasmosis serology revealed raised IgG antibody levels of $325 \mathrm{IU} / \mathrm{ml}$.

He was treated with mannitol, trimethoprim (160mg)/ sulfamethoxazole $(800 \mathrm{mg})$ thrice daily, pyrimethamine $(25 \mathrm{mg}) /$ sulfadoxine $(500 \mathrm{mg})$ thrice daily ,folinic acid and anticonvulsants. His symptoms such as confusion, headache improved gradually within one week of admission. After two weeks repeat MRI of brain was done which showed significant resolution of the lesions (Figure 2).

Repeat MRI of brain after 2 weeks showing significant resolution of the lesions.

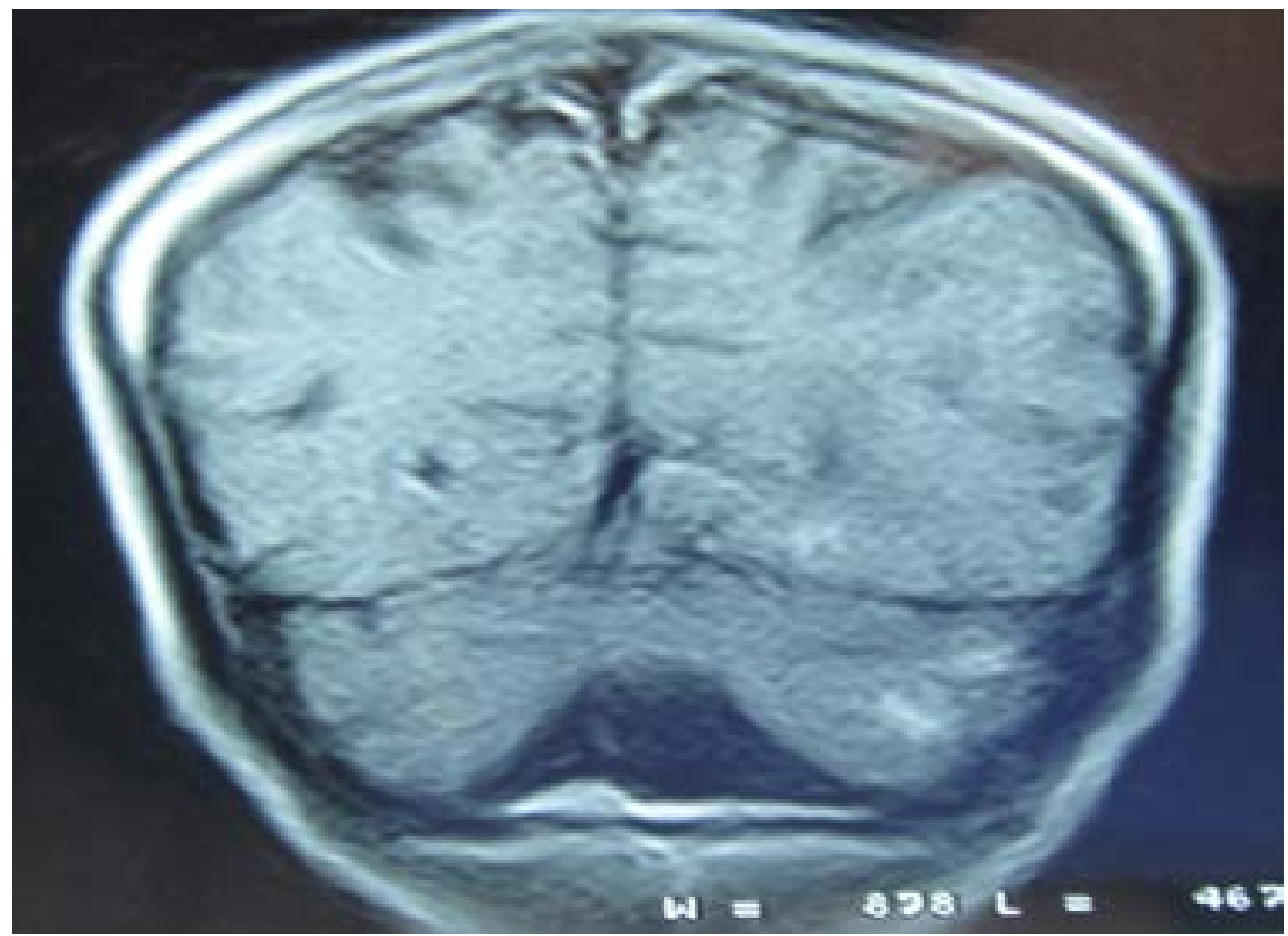


He was treated for three weeks. Antiretroviral drugs were started (Tenofovir (300mg), Emtricitabine (200mg) and Efavirenz $(600 \mathrm{mg}$ ) once daily. Patient was discharged from hospital in an ambulatory state. He was advised to continue antiretroviral drugs, anticonvulsants and trimethoprim/sulfamethoxazole (prophylaxis) .He has been on regular follow up for the past thirty months.

\section{Discussion}

Neurologic manifestations are frequent in patients with human immunodeficiency virus (HIV) infection.

Toxoplasmosis is caused by Toxoplasma gondii. Cats are definitive hosts. They excrete parasite's oocysts in their faeces. Transmission occurs due to ingestion of either oocysts from contaminated soil, food or water or from undercooked meat. The major clinical features of cerebral toxoplasmosis are headache, fever, altered sensorium, seizures and focal neurological defecits ${ }^{2}$

The classical radiological pictures of Toxoplasmosis are multiple parenchymal lesions, with ring enhancement, which are generally localized at the corticomedullary junction or basal ganglia. On MRI these lesions appear hypointense on T1 weighted images with up to $70 \%$ showing ring enhancement with contrast ${ }^{3}$. Toxoplasmosis may just present with hydrocephalus in the absence of parenchymal lesions ${ }^{4}$. Cerebral toxoplasmosis and primary cerebral lymphoma are the common causes of ring enhancing lesions of central nervous system in patients with AIDS. However, it is often difficult to distinguish between these two conditions clinically and radiographically ${ }^{5}$.

Neurocysticercosis must be included in the differential diagnosis of neurologic infections in HIV patients in endemic populations ${ }^{6}$. Other conditions that can cause ring enhancing lesions are tuberculomas, gliomas, metastases and abscesses ${ }^{7}$.

The definitive diagnostic procedure is brain biopsy ${ }^{8}$. Brain biopsy should only be considered in patients with negative toxoplasmosis serology and who do not respond to treatment ${ }^{9}$. Negative serology for antitoxoplasma IgG antibodies should prompt the clinician to consider an alternative diagnosis ${ }^{10}$. Molecular diagnosis (conventional PCR and other types of PCR) is also an important tool to detect toxoplasmosis ${ }^{11,12}$. These new tools are not easily available for clinicians in developing countries.
Pyrimethamine (200-mg oral loading dose, followed by $50-75 \mathrm{mg} /$ day orally) sulfadiazine (1500 mg 4 times daily) or Trimethoprim (5 mg/ $\mathrm{kg}$ ) / sulfamethoxazole $(25 \mathrm{mg} / \mathrm{kg}$ ) twice daily are the drugs that are used to treat toxoplasmosis ${ }^{10}$. In our centre, we use trimethop$\mathrm{rim} /$ sulfamethoxazole and pyrimethamine /sulfadoxine to treat AIDS- associated cerebral toxoplasmosis as sulfadiazine is not available in our state. Trimethoprim/ sulfamethoxazole is an alternative treatment for toxoplasma encephalitis because it is inexpensive and as effective as pyrimethamine-sulfadiazine ${ }^{13}$. In toxoplasmosis radiological improvement is seen in most of the patients after two to three weeks of treatment ${ }^{14}$. MRI brain was repeated in our patient after 2 weeks which showed resolution of the lesions.At least three weeks of treatment is needed for toxoplasmosis.

Our patient presented with altered sensorium, hemiparesis and MRI brain revealed multiple lesions in bilateral supratentorial and infratentorial neuroparenchyma. Early empirical antitoxoplasmosis treatment based on MRI was started and the patient improved.

\section{Conclusion}

Cerebral toxoplasmosis should be considered in the differential diagnosis of multiple neuroparenchymal lesions in individuals who present with neurological deficits. Once cerebral toxoplasmosis is suspected, treatment should be started empirically pending the confirmation of the diagnosis. Imaging studies of the brain along with serology play an important role in the diagnosis of toxoplasmosis.

\section{References}

1. Doraiswamy V, Vaswani RK, Lahiri KR, Kondekar SS. Neurotoxoplasmosis mimicking intracranial tuberculoma. J Postgrad Med. 2010 ;56(1):31 -4.

2. Luma HN, Tchaleu BC, Mapoure YN, Temfack E, Doualla MS, Halle MP, et al. Toxoplasma encephalitis in HIV/AIDS patients admitted to the Douala general hospital between 2004 and 2009: a cross sectional study. BMC Res Notes. 2013 ;6(1):146 PubMed .

3. Gupta A, Raja A, Mahadevan A, Shankar S K. Toxoplasma granuloma of brainstem: A rare case. Neurol India 2008;56:189-91 PubMed

4. Basavaprabhu A, Soundarya M, Deepak M, Satish R. CNS Toxoplasmosis Presenting with Obstructive Hydrocephalus in Patients of Retroviral Disease- A Case Series. Med J Malaysia 2012; 67:214-16 PubMed . 
5. Schroeder PC, Post MJ, Oschatz E, Stadler A, Bruce-Gregorios J, Thurnher MM. Analysis of the utility of diffusion-weighted MRI and apparent diffusion coefficient values in distinguishing central nervous system toxoplasmosis from lymphoma. Neuroradiology 2006; 48: 715- PubMed ;20.

6. Serpa JA, Moran A, Goodman JC, Giordano TP, White AC Jr. Neurocysticercosis in the HIV era: a case report and review of the literature. Am J Trop Med Hyg. 2007; 77:113-7 PubMed .

7. Garg R K, Sinha M K. Multiple ring-enhancing lesions of the brain. J Postgrad Med 2010;56:307-16

8. Yoganathan K. A "brain tumor" in an intravenous drug abuser. International Journal of General Medicine 2009;2:73-75.

9. Mathews C, Barba D, Fullerton SC. Early biopsy versus empiric treatment with delayed biopsy of nonresponders in suspected HIV-associated cerebral toxoplasmosis: a decision analysis. AIDS. 1995;9:1243-50. 10. Jayawardena S, Singh S, Burzyantseva O, Clarke H:
Cerebral toxoplasmosis in adult patients with HIV infection. Clin Med J Resid Hosp Physician 2008 ;44(7):1724.

11. Robert-Gangneux F, Dardé M-L. Epidemiology of and Diagnostic Strategies for Toxoplasmosis. Clinical Microbiology Reviews 2012;25(2):264 PubMed -296.

12. Sukthana Y, Mahittikorn A, Wickert H, Tansuphaswasdikul S. A promising diagnostic toolfor toxoplasmic encephalitis: tachyzoite/bradyzoite stage-specific RTPCR. Int J Infect Dis. 2012 ;16(4): PubMed e279-84.

13. Béraud G, Pierre-François S, Foltzer A, Abel S, Liautaud B, Smadja D, et al. Cotrimoxazole for treatment of cerebral toxoplasmosis: an observational cohort study during 1994-2006. Am J Trop Med Hyg. 2009;80(4):583 PubMed -7.

14. Luft BJ, Hafner R, Korzun AH, Leport C, Antoniskis D, Bosler EM, et al. Toxoplasmic encephalitis inpatients with the acquired immunodeficiency syndrome. Members of the ACTG 077p/ANRS 009 Study Team. N Engl J Med 1993; 329:995-1000 PubMed . 